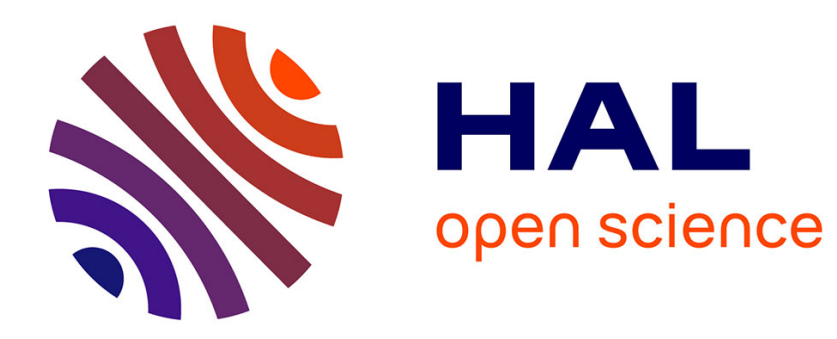

\title{
Surface Conduction Model for Fractal Porous Media
}

\author{
Hongtao Wang, André Revil
}

\section{To cite this version:}

Hongtao Wang, André Revil. Surface Conduction Model for Fractal Porous Media. Geophysical Research Letters, 2020, 47, 10.1029/2020gl087553 . hal-03005826

\section{HAL Id: hal-03005826 \\ https://hal.science/hal-03005826}

Submitted on 14 Nov 2020

HAL is a multi-disciplinary open access archive for the deposit and dissemination of scientific research documents, whether they are published or not. The documents may come from teaching and research institutions in France or abroad, or from public or private research centers.
L'archive ouverte pluridisciplinaire HAL, est destinée au dépôt et à la diffusion de documents scientifiques de niveau recherche, publiés ou non, émanant des établissements d'enseignement et de recherche français ou étrangers, des laboratoires publics ou privés. 


\section{Geophysical Research Letters}

\author{
RESEARCH LETTER \\ 10.1029/2020GL087553 \\ Key Points: \\ - We developed a power law \\ relationship between surface \\ conductivity and specific surface \\ area \\ - The exponent of this relationship is \\ related to the porosity/cementation \\ exponent and to the fractal \\ dimension of the pore network \\ - The electrical properties entering \\ the newly developed conductivity \\ model can be used to predict \\ permeability
}

Supporting Information:

- Supporting Information S1

Correspondence to:

A. Revil,

andre.revil@univ-smb.fr

Citation:

Wang, H., \& Revil, A. (2020). Surface conduction model for fractal porous media. Geophysical Research Letters, 47, e2020GL087553. https://doi.org/ 10.1029/2020GL087553

Received 16 FEB 2020

Accepted 20 APR 2020

Accepted article online 29 APR 2020

(C)2020. American Geophysical Union. All Rights Reserved.

\section{Surface Conduction Model for Fractal Porous Media}

\author{
Hongtao Wang ${ }^{1}$ (D) and André Revil ${ }^{2}$ (iD) \\ ${ }^{1}$ State Key Laboratory of Oil and Gas Reservoir Geology and Exploitation (Chengdu University of Technology), College of \\ Geophysics, Chengdu University of Technology, Chengdu, China, ${ }^{2}$ University Grenoble Alpes, University Savoie \\ Mont-Blanc, CNRS, UMR CNRS 5204, EDYTEM, Le Bourget du Lac, France
}

Abstract The electrical conductivity of natural water-saturated porous materials has two contributions, one associated with conduction in the pore network and a second one associated with the electrical double layer coating the surface of the mineral grains, and called surface conductivity. We model the effect of material texture on surface conductivity based on fractal theory. We demonstrate that surface conductivity obeys a power-law dependence (that we called surface Archie's law) on the specific surface area. The power exponent is theoretically related to the porosity exponent entering Archie's law and to the fractal dimension of the pore network. A permeability model is derived by combining the new surface Archie's law and the Kozeny-Carman model. We show that our model is consistent with both numerical finite element simulations on synthetic porous media and experimental data.

Plain Language Summary Electrical conductivity tomography is a powerful method to characterize porous media in the realm of geophysics. In absence of conduction mechanisms on the surface of the grains, the conductivity of a porous material is connected to porosity through a relationship known as Archie's law. Surface conductivity associated with conduction along the electrical double layer coating the surface of the grains is related to the specific surface area of the material. Based on fractal theory, we develop a new power law relationship between the surface conductivity and the specific surface area. This theory can improve our ability to interpret electrical conductivity data at various scales and to connect electrical properties to permeability.

\section{Introduction}

Archie (1942) proposed an empirical power function connecting the rock bulk conductivity $\sigma\left(\mathrm{S} \mathrm{m}^{-1}\right)$ to the (connected) porosity $\phi$ (dimensionless) by $\sigma=\sigma_{\mathrm{w}} / F=\phi^{\mathrm{m}} \sigma_{\mathrm{w}}$ where $\sigma_{\mathrm{w}}\left(\mathrm{S} \mathrm{m}^{-1}\right)$ is the conductivity of the pore fluid, $F$ (dimensionless) denotes formation factor and $m$ (dimensionless) the porosity or cementation exponent. Winsauer et al. (1952) generalized Archie's law to $F=a \phi^{-\mathrm{m}}$ where $a$ is a constant (see discussion in Glover, 2016). This generalized Archie's law can be sometimes derived from first principles, e.g., for instance using the differential effective medium (DEM) approach on granular media (Mendelson \& Cohen, 1982). The investigation of the physical basis and possible extensions for Archie's law has been the subjects of numerous studies (e.g., Glover et al., 2000; Hunt, 2004; Mendelson \& Cohen, 1982; Sen et al., 1981; Wong et al., 1984; Yue, 2019). Previous researchers have demonstrated that fractal theory is an efficient tool to characterize the textures of geological materials (especially soils and sedimentary rocks), and based on these observations, Archie's law can be derived from fractal theory (e.g., Katz \& Thompson, 1985; Wang \& Liu, 2017; Wei et al., 2015).

In addition to the bulk conductivity associated with conduction in the bulk pore network, an additional contribution called surface conductivity is important in clayey formations (Patnode \& Wyllie, 1950). Surface conduction is contributed by the counterions in the electrical double layer adjacent to the mineral surface. The model of Waxman and Smits (1968) leads to the following expression for the surface conductivity $\sigma_{\mathrm{S}}=\widehat{B}$ $Q_{\mathrm{V}} / F$ where the term $\widehat{B}$ denotes the apparent mobility of the counterions surrounding the mineral grains and $Q_{\mathrm{V}}$ denotes the excess of counterions per unit pore volume. In the so-called high salinity asymptotic limits defined by Johnson et al. (1986), surface conductivity increases linearly with the specific surface conductance $\Sigma_{S}$ (in $S$ ) describing the excess conductivity in the vicinity of the surface of the grains. Johnson et al. (1986) obtained the following high-salinity asymptotic conductivity equation 


$$
\sigma=\frac{\sigma_{\mathrm{w}}}{F}+\sigma_{\mathrm{S}}=\frac{\sigma_{\mathrm{w}}}{F}+\frac{\Sigma_{\mathrm{S}}}{F_{\mathrm{S}}}
$$

where $\sigma_{\mathrm{S}}=\Sigma_{\mathrm{S}} / F_{\mathrm{S}}, F_{\mathrm{S}}$ is a parameter only related to the texture and called surface formation factor below. Effective media models describe the conductivity of a two-phase system in which one phase exists as inclusion (Sen et al., 1981). They require however specific assumptions regarding the microgeometry of the porous material including the presence of inclusions.

Our goal is to develop an alternative theoretical model for $F_{\mathrm{S}}$ based on fractal theory. Our starting assumption is that the proper geometry responsible for the validity of Archie's law implies that it can be used to model surface conductivity as well. Therefore, we select fractal geometry because it allows the description of a complex rock microstructure by a small number of parameters (Anovitz \& Cole, 2015; Thompson et al., 1987) and because the classical Archie's law can be deduced within that framework (Cai et al., 2017). Furthermore, modeling of surface conduction can be used to predict the permeability of rocks (Thompson et al., 1987). Just as $F$ can be used to estimate the tortuosity of fluid flow in the connected pore space, $F_{\mathrm{S}}$ can be useful in providing the hydraulic radius (Bernabé \& Revil, 1995; Johnson et al., 1986; Revil \& Cathles, 1999).

In the following, we first derive a relationship between $F_{\mathrm{S}}$ and the specific surface area (surface area of the solid phase per medium volume) based on fractal geometry. Following this relationship, a new revised permeability model is also given. Numerical finite element simulations (e.g., Shabro et al., 2014) and core measurements are then used to test the predictions of our model using synthetic fractal porous media and natural sandstones.

\section{Theory}

\subsection{Parameter Definition}

Consider a cubic representative elemental volume (REV) of size $L(\mathrm{~m})$ saturated with brine and where the pore-grain interface is characterized by a thin conductive layer. An electrical potential difference $\Delta \Psi(V)$ is applied on two opposite faces of the REV, while the others have insulating boundary conditions. At high salinity, the local electrical field $e_{\mathrm{b}}$ in pore space $\left(\mathrm{Vm}^{-1}\right)$ is controlled by the distribution of the pore brine. The total Joule dissipation of current flow in such a system equals the energy generated in the pore space and in the surface layer (Johnson et al., 1986):

$$
\sigma\left(\frac{\Delta \Psi}{L}\right)^{2}=\frac{\sigma_{\mathrm{w}}}{V} \int_{V_{\mathrm{p}}}\left|\mathrm{e}_{\mathrm{b}}\right|^{2} d V_{\mathrm{p}}+\frac{\Sigma_{\mathrm{S}}}{V} \int_{\mathrm{S}}\left|\mathrm{e}_{\mathrm{b}}\right|^{2} d S .
$$

From equations 1 and 2, the bulk and surface formation factors are defined as

$$
\frac{1}{F}=\frac{1}{V} \int_{V_{\mathrm{p}}}\left|\widehat{\mathrm{e}}_{\mathrm{b}}\right|^{2} d V_{\mathrm{p}}
$$

and

$$
\frac{1}{F_{\mathrm{S}}}=\frac{1}{V} \int_{\mathrm{S}}\left|\widehat{\mathrm{e}}_{\mathrm{b}}\right|^{2} d S
$$

in which $\widehat{\mathrm{e}}_{b}=e_{b} L / \Delta \Psi$ is the normalized local electrical field. The definitions of $F$ and $F_{\mathrm{S}}(\mathrm{m})$ reveal that the former is scale independent, while the latter is scale dependent. This means when a REV described above is compressed by a factor $p(>1), 1 / F$ does not change while $1 / F_{\mathrm{S}}$ changes by a factor $p / F_{\mathrm{S}}$. This theorem will be used in the following.

\subsection{Archie's Law for Bulk Electrical Conduction}

We assume that the REV has a fractal pore space. To simplify the analysis, we assume a Sierpinski carpet as a reference model (Allouche \& Shallit, 2003). As a deterministic fractal, the Sierpinski carpet (SC) can be constructed iteratively to build a type of fractal porous material. We start by dividing a square of length $L$ into $p \times p(p=3)$ congruent subsquares and then removing $p \times p-N(N=8)$ of them. At the next iteration, 
the previous operation is applied again to each of the remaining $N$ subsquares. This procedure is repeated until reaching the minimum fractal pore size or the expected porosity. The geometry obtained at the first iteration stage is called a generator. The pore fractal dimension $D_{f}$ is calculated as $D_{f}=\ln (N) / \ln (p)($ e.g., Peitgen \& Saupe, 1988).

Our task is to formulate the dependence of conductivity on porosity and specific surface area. We first follow the approach used by Wang and Liu (2017) to derive the classical Archie's law. In this derivation, only self-similarity is a key requirement, so the result can be expanded to any self-similar fractals. Obviously, the porosity of the SC at stage $i$ (SC $i$ ) is $\phi_{i}=\phi_{1}^{i}$. The porosity of the generator is $\phi_{1}=N / p^{D}$, where $D$ is the Euclidean dimension of the embedding space ( $D=2$ or 3). Denote the conductivity of SC $i$ as $\sigma_{i}$ $\left(\sigma_{0}=\sigma_{\mathrm{w}}\right)$. Notice that $\sigma_{i}$ does not change when SC $i$ is scaled larger or smaller and SC $i$ is composed of $N$ SC $i-1$ around the center solid grain. So $\sigma_{i}$ is a function of the conductivity and geometrical arrangement $G$ of the NSC $i-1: \sigma_{i}=f\left(\sigma_{i-1}, G\right)=\sigma_{i-1} f(1, G)$. Because of the self-similarity property of fractal, $G$ does not change with stage $i$. After setting $i=1$, we have $f(1, G)=\sigma_{1} / \sigma_{\mathrm{w}}$. The final expression of $\sigma_{i}$ is $\sigma_{i}=\sigma_{\mathrm{w}} f(1, G)^{i}$. By combining their expressions, the relation between $\sigma_{i}$ and $\phi_{i}$ is written as $\sigma_{i}=\sigma_{\mathrm{w}} \phi_{i}^{\mathrm{m}}$ with $m=\ln (f(1, G)) / \ln$ $\left(\phi_{1}\right)$. So Archie's law is derived simply and implies that the properties of the generator play a significant role in the conductivity of fractal porous media. This is not surprising because the generator represents the basic starting geometry of the fractal.

\subsection{An Interfacial Archie's Law for Surface Conductivity}

We consider now the role of surface conduction. We consider an initial stage, stage 0 , containing $100 \%$ brine, and grains are progressively added to this background. With the increase of fractal stage of the SC, more and more grains are added into the pore volume, and therefore, the inner surface area of the porous material increases. As SC $i$ is composed of $N$ SC $i-1$, the total inner surface of SC $i$ can be separated into two parts: one includes the surface of SC 1; another includes the surface of the N SC $i-1$ after being downscaled by a factor $p$. So the specific surface area $S_{\mathrm{V}}\left(\mathrm{m}^{-1}\right)$ of $\mathrm{SC} i$ is

$$
\begin{aligned}
\left(S_{\mathrm{V}}\right)_{i} & =\frac{N}{p^{D-1}}\left(S_{\mathrm{V}}\right)_{i-1}+\left(S_{\mathrm{V}}\right)_{1}=\sum_{j=0}^{i-1}\left(S_{\mathrm{V}}\right)_{1}\left(\frac{N}{p^{D-1}}\right)^{j} \\
\left(S_{\mathrm{V}}\right)_{i} & =\left(S_{\mathrm{V}}\right)_{1} \frac{1-\left(N / p^{D-1}\right)^{i}}{1-N / p^{D-1}} \approx\left(S_{\mathrm{V}}\right)_{1} \frac{\left(N / p^{D-1}\right)^{i}}{N / p^{D-1}-1}
\end{aligned}
$$

In the last derivation process of equation $6,\left(N / p^{D-1}\right)^{i}$ is larger than one, which can be checked through numerical modeling. This corresponds to $D_{f}>D-1$, a requirement for porous materials to keep the pore space fully connected in all directions (for instance, we have $D_{f}>2.5$ for the sandstones studied by Thompson et al., 1987). So the assumption in equation 6 is reasonable.

The conductivity of the fractal is also contributed by electrical conduction in two parts: one includes the surface of the SC 1 ; another includes the pore and surface of the NSC $i-1$ after scaled by the factor $p$. Therefore, the conductivity of the fractal becomes

$$
\begin{gathered}
\sigma_{i}=\sigma_{i-1} f(1, G)+\frac{\Sigma_{\mathrm{S}}}{\left(F_{\mathrm{S}}\right)_{1}}=\left(\frac{\sigma_{\mathrm{w}}}{F_{i-1}}+\frac{p \Sigma_{\mathrm{S}}}{\left(F_{\mathrm{S}}\right)_{i-1}}\right) f(1, G)+\frac{\Sigma_{\mathrm{S}}}{\left(F_{\mathrm{S}}\right)_{1}} \\
\sigma_{i}=\frac{\sigma_{\mathrm{w}}}{F_{i-1}} f(1, G)+\left(\frac{p f(1, G)}{\left(F_{\mathrm{S}}\right)_{i-1}}+\frac{1}{\left(F_{\mathrm{S}}\right)_{1}}\right) \Sigma_{\mathrm{S}}
\end{gathered}
$$

Thus, $1 / F_{\mathrm{S}}$ can be expressed as

$$
\begin{gathered}
\frac{1}{\left(F_{\mathrm{S}}\right)_{i}}=\sum_{j=0}^{i-1} \frac{1}{\left(F_{\mathrm{S}}\right)_{1}}(p f(1, G))^{j} \\
\frac{1}{\left(F_{\mathrm{S}}\right)_{i}}=\frac{1}{\left(F_{\mathrm{S}}\right)_{1}} \frac{1-(p f(1, G))^{i}}{1-p f(1, G)} \approx \frac{1}{\left(F_{\mathrm{S}}\right)_{1}} \frac{(p f(1, G))^{i}}{p f(1, G)-1} .
\end{gathered}
$$


The last derivation process assumes $(p f(1, G))^{i}$ is notably larger than one. With the definitions of $m$ and $D_{f}$, it is easy to find that this assumption is equivalent to $D_{f}>D-m^{-1}$. This requirement is therefore stronger than the one used to establish the validity of equation 6 . From equations 6 and 10, the relationship between $F_{\mathrm{S}}$ and $S_{\mathrm{V}}$ (removing the subscript $i$ ) can be derived

$$
\begin{gathered}
\frac{1 / F_{\mathrm{S}}}{1 /\left(F_{\mathrm{S}}\right)_{1}}=\left[\frac{S_{\mathrm{V}}}{\left(S_{\mathrm{V}}\right)_{1}}\right]^{m_{\mathrm{S}}}, \\
m_{\mathrm{S}} \equiv \frac{\ln (p f(1, G))}{\ln \left(N / p^{D-1}\right)}=m+\frac{m-1}{D-D_{f}-1} .
\end{gathered}
$$

Equation 12 is the one of the two fundamental equations derived in this paper. As $D_{f}$ is assumed to be larger than $D-m^{-1}$, we can derive from equation 12 that $m_{\mathrm{S}}$ is necessarily smaller than 1 , while $m>1$. Equation 11 implies that the ratio of $1 / F_{\mathrm{S}}$ of a fractal at any two stages is a power function of $S_{\mathrm{V}}$. We introduce a parameter $a_{\mathrm{S}}$, which is defined to be the value of $F_{\mathrm{S}}$ when $S_{\mathrm{V}}=1 \mathrm{~m}^{-1}$. Then, equation 11 is transformed to

$$
\frac{1}{F_{\mathrm{S}}}=\frac{1}{a_{\mathrm{S}}} S_{\mathrm{V}}^{m_{\mathrm{S}}}
$$

We call equation (13) a "surface Archie's law" and $a_{\mathrm{S}}$ a constant coefficient in this law and $m_{\mathrm{S}}$ the specific surface area exponent. The above derivation is based on fractals like SC in which grains are suspended in fluid. For compacted material, the effect of grain contact should be taken into consideration. The derivation of surface Archie's law in this case is slightly different (Appendix A) but yields the same result.

\subsection{Effective Hydraulic Radius and Permeability}

The classical Kozeny-Carman (KC) model is extensively used to predict permeability, and one of its variants can be written as (Walsh \& Brace, 1984)

$$
k=\frac{1}{b F}\left(\frac{V_{\mathrm{p}}}{S}\right)^{2},
$$

where $b$ is a constant and equal to 2 for circular tubes. The use of the formation factor $F$ corrects for the influence of the tortuosity of the pore space. The ratio of pore volume to pore surface area, $V_{\mathrm{p}} / S(\mathrm{~m})$, represents the pore radius and is treated as hydraulic radius. Another pore scale parameter, the effective electrical pore radius $\Lambda(\mathrm{m})$, can be defined as (Johnson et al., 1986)

$$
\frac{2}{\Lambda}=\frac{F}{F_{\mathrm{S}}}=\frac{\int_{S}\left|\widehat{\mathrm{e}}_{\mathrm{b}}\right|^{2} d S}{\int_{V_{\mathrm{p}}}\left|\widehat{\mathrm{e}}_{\mathrm{b}}\right|^{2} d V_{\mathrm{p}}}
$$

The quantity $\Lambda / 2$ has also been used to replace $V_{\mathrm{p}} / S$ in equation 14 to improve permeability prediction (e.g., Bernabé \& Bruderer, 1998; Revil \& Cathles, 1999). As $\Lambda$ can be calculated with surface Archies law, a revised KC-type model is simply derived

$$
k=\frac{1}{b F^{3}}\left(\frac{S_{\mathrm{V}}^{m_{\mathrm{S}}}}{a_{\mathrm{S}}}\right)^{-2}
$$

\section{Results and Discussion}

In this section, we test the derived relationships with finite element simulations and core conductivity measurements. The numerical simulations are based on three fractals: the SC, the Menger sponge (MS), and the second fractal (RTB) of Roy and Tarafdar (1997). The finite element simulation is conducted with the software Comsol. Each fractal has a side length of $1 \mathrm{~mm}$. After the simulation of the electrical field in each fractal, the parameters, $F$ and $F_{\mathrm{S}}$, are calculated from equations 3 and 4 , respectively. 


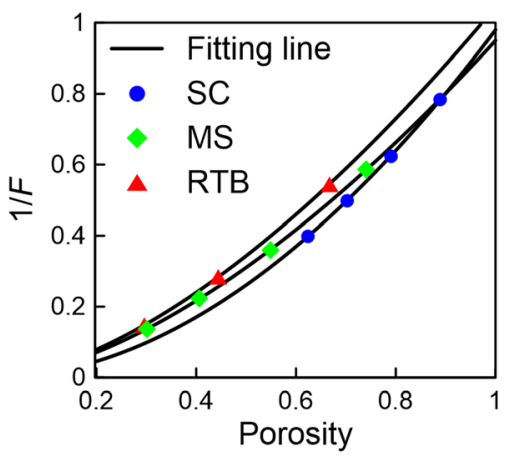

C

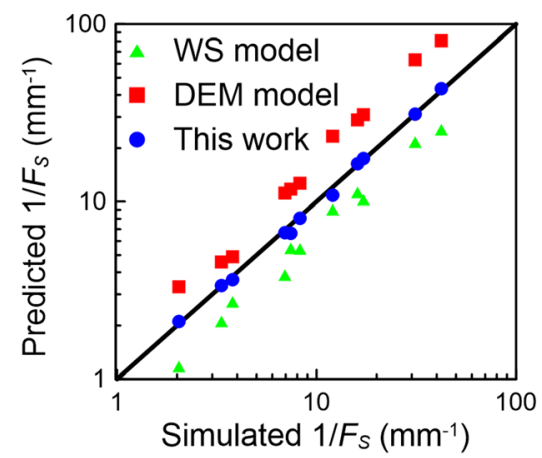

b

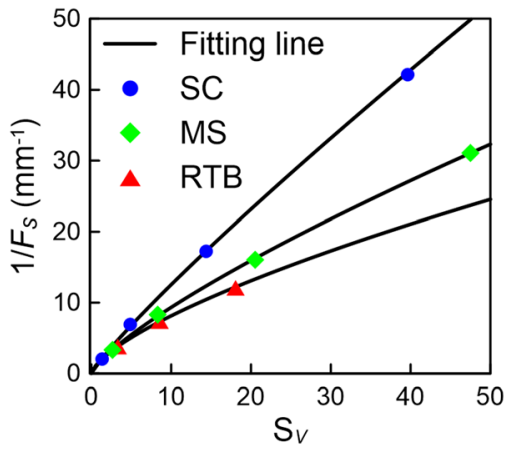

d
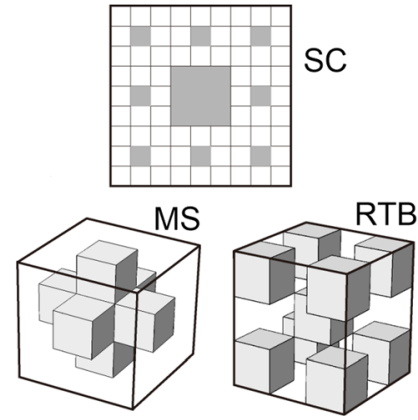

Figure 1. Simulation results and comparison of model predictions for three fractals: Sierpinski carpet (SC), Menger sponge (MS), and RTB from Roy and Tarafdar (1997). (a) Relationship between $1 / F$ and porosity. (b) Relationship between $1 / F_{\mathrm{S}}$ and $S_{\mathrm{V}}$ (c) Comparison between simulated $1 / F_{\mathrm{S}}$ and the predictions of Waxman-Smits (WS) model, differential effective medium (DEM) model and this work. (d) Diagrams of the three fractals: the SC at stage 2 and generators of the MS and RTB. The gray color represents grains. Archie's law and surface Archie's law are used to fit the data in (a) and (b), respectively, and the fitting parameter values are listed in Table 1.

The simulation results of $F$ and $F_{\mathrm{S}}$ of the three fractals are demonstrated in Figure 1. It shows that $F$ and $\phi$ satisfy Archie's law, and $F_{\mathrm{S}}$ and $S_{\mathrm{V}}$ satisfy the surface Archie's law. The fitting values of model parameters are listed in Table 1 , and the coefficient of determination $\left(R^{2}\right)$ of every fitting is at least 0.999 . Note that the fitting curves are lower convex and upper convex in Figures $1 \mathrm{a}$ and $1 \mathrm{~b}$, respectively, meaning that $m>1$ and $m_{S}<1$. The $m_{\mathrm{S}}$ calculated from $m$ and $D_{f}$ with equation 12 is very close to the fitting values (Table 1 ). Using the calculated $m_{\mathrm{S}}$ and setting $a_{\mathrm{S}}$ equal the fitting values, $1 / F_{\mathrm{S}}$ can be predicted with equation 13 . Figure 1c shows that the predictions agree well with the simulations.

Among the fractals, the 2D SC generally has the smallest pore conduction but largest surface conduction (Figure 1). We present the predictions of $1 / F_{\mathrm{S}}$ using both the Waxman and Smits (1968) model and the DEM model of Bussian (1983) in Figure 1c. Waxman-Smits model underestimates $1 / F_{\mathrm{S}}$, implying that the surface is actually less tortuous than pore space in these three fractals. By contrast, the DEM model tends to overestimate $1 / F_{\mathrm{S}}$. For the Waxman-Smits model, the pore space is composed of capillaries. While in the DEM model, the grains correspond to the inclusions embedded in the pore space. This two contrasting textures may explain why the two models have opposite behaviors in Figure 1c.

The status of $a_{\mathrm{S}}$ in equation 13 is similar to $a$ in Archie's law; the latter is usually chosen to be 1 and equals the formation factor when $\phi=1$. From the definition of $a_{\mathrm{S}}$ (equals to $F_{\mathrm{s}}$ when $S_{\mathrm{V}}=1\left(\mathrm{~m}^{-1}\right)$ ), we cannot find any reason to set $a_{\mathrm{S}}$ to some fixed value. But we emphasis that it is not an accident that the fitting values of different $a_{\mathrm{S}}$ in Table 1 are close to each other. The same feeling can also be directly obtained by observing that the three curves in Figure $1 \mathrm{~b}$ converges to the origin as $S_{\mathrm{v}}$ decreases to 0 . Consider such a situation that when putting a spherical grain into a cubic pore space of unit length, the porosity is about 0.9 if $S_{\mathrm{V}}=1$. Thus, we claim that $S_{\mathrm{V}}$ of 1 only represents a small fraction of the surface. This fact explains partly why the value of $a_{\mathrm{S}}$ is stable. 
Table 1

Petrophysical parameters of the three fractals in study. The values of $a, m$, $a_{S}$, and $m_{S}$ are obtained through fitting the simulation data

\begin{tabular}{llllcll}
\hline Fractals & $D_{f}$ & $a$ & $m$ & $\mathrm{~S}(\mathrm{~mm})$ & $m_{\mathrm{S}}$ & $m_{\mathrm{S}}{ }^{*}$ \\
\hline SC & 1.893 & 0.980 & 1.910 & 1.629 & 0.886 & 0.891 \\
MS & 2.727 & 0.950 & 1.613 & 1.575 & 0.772 & 0.769 \\
RTA & 2.631 & 1.044 & 1.606 & 1.672 & 0.687 & 0.646 \\
\hline
\end{tabular}

Note. The values of $m_{\mathrm{S}}{ }^{*}$ is calculated through equation 12 . SC: Sierpinski carpet; MS: Menger sponge; RTB: fractal B in Roy and Tarafdar (1997).
Two groups of conductivity measurements made on saturated sandstones from Waxman and Smits (1968) and Vinegar and Waxman (1984) are used to test the model on real rocks. The measurements were conducted at different salinities. The conductivities in high-salinity region are used to determine $F$ and $F_{\mathrm{S}}$. Under the assumption that the counterions distribute uniformly on the surface, the counterion content per volume of the material $Q_{\mathrm{V}} \phi\left(C E C_{t}\right)$ is proportional to $S_{\mathrm{V}}$ and (equals to $\rho_{\mathrm{g}}(1-\phi)$ CEC where $\rho_{\mathrm{g}}$ denotes the mass density of the grains and CEC the cation exchange capacity). Thus, the surface Archie's law is transformed to

$$
\frac{1}{F_{\mathrm{S}}}=\frac{1}{a_{\mathrm{S}}}\left(\frac{C E C_{t}}{Q_{\mathrm{S}}}\right)^{m_{\mathrm{S}}} \sim C E C_{t}^{m_{\mathrm{S}}}
$$

where $Q_{\mathrm{S}}\left(\mathrm{C} \mathrm{m}^{-2}\right)$ denotes the surface charge density of counterions, which is typical equal to 2 to 4 elementary charges per $\mathrm{nm}^{2}$ (see Revil et al., 2017). The data $\sigma_{\mathrm{S}}$ versus $C E C_{t}$ are plotted in Figure 2 . It is shown these data can be well fitted with equation 17. The fitting values of $m_{\mathrm{s}}$ for both data sets are smaller than 1 , and the coefficient values (0.44 and 0.47) are close to each other (similarly the values of $a_{\mathrm{S}}$ for the three investigated fractals were in a very narrow range).

In the following, we present the application of the revised $\mathrm{KC}$ model, equation 16, to core data. Petrophysical properties of 24 shaly sandstones were measured by Zhang and Weller (2014), including permeability, conductivity (in high salinity), specific surface area, porosity, and pore fractal dimension. So the parameter $m_{\mathrm{S}}$ can be directly calculated. The coefficient $a_{\mathrm{S}}$ is set to $1.60 \mu \mathrm{m}$, the average fitting value of the three fractals (Table 1). We compare in Figure 3 the core permeabilities predicted by the revised $\mathrm{KC}$ model and the classical KC model (with $b=2$ ) with the core permeability measurements. The prediction of the revised model is closer to the core measurements than the classical $\mathrm{KC}$ model. The root mean square error of $\log _{10}(k)$ of the revised model is 0.373 , smaller than that of the $\mathrm{KC}$ model, 0.487.

Within the framework of fractals, our theory offers new relationship between the bulk conductivity, the surface conductivity, the permeability, and the texture of rocks. The surface Archie's law is comparable to the classical Archie's law in a very fundamental way. Both of them express the same concept: the conductivity has power dependence on the amount of available ions for electromigration (i.e., the porosity and surface) in a porous medium characterized by a fractal dimension. It is interesting that the surface power-law exponent can be calculated from $m$, providing $D_{f}$ is known. It is well known that $D_{f}$ contains the information of pore volume, rather than pore connectivity and tortuosity. Our theory reveals that the two parameters $m$ and $D_{f}$ are enough to determine the effect of texture on the surface conduction at the high salinity asymptotic behavior corresponding to the validity of equation 1.

Though the surface Archie's law is derived mostly for surface conduction in shaly sandstones, the revised KC model following this law seems to be applicable to the cases where the surface conduction is not significant.

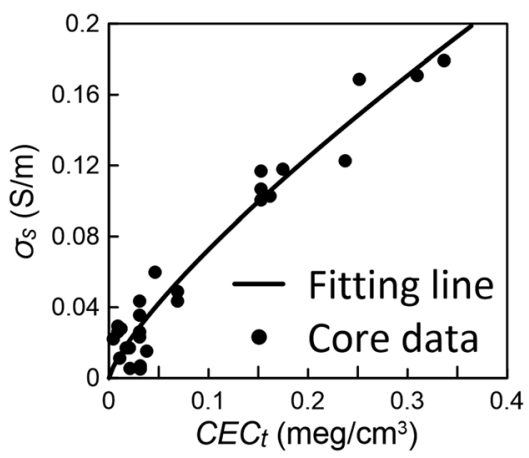

b

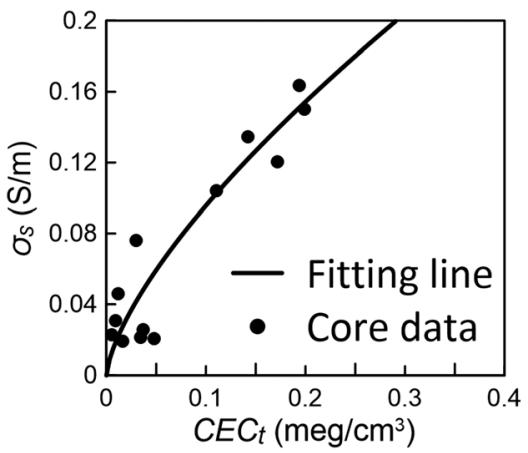

Figure 2. Relationship between $\sigma_{\mathrm{S}}$ and $C E C_{t}$. (a) Data from Waxman and Smits (1968). (b) Data from Vinegar and Waxman (1984). These data are fitted with surface Archie's law. The fitting results in (a): $m_{\mathrm{S}}=0.78$, coefficient $a_{\mathrm{S}}=0.44,=0.94 ;$ and in (b): $m_{\mathrm{S}}=0.69$, coefficient $a_{\mathrm{S}}=0.47, R^{2}=0.85$. 
a

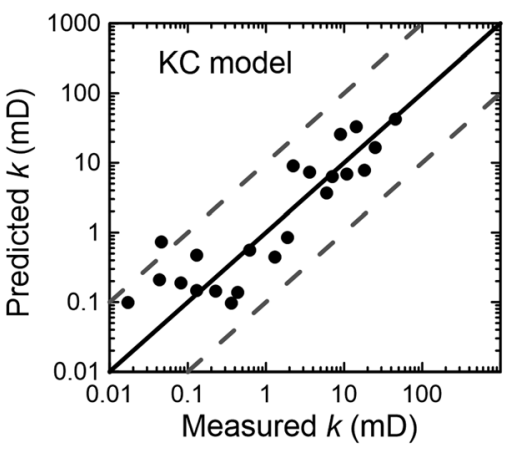

b

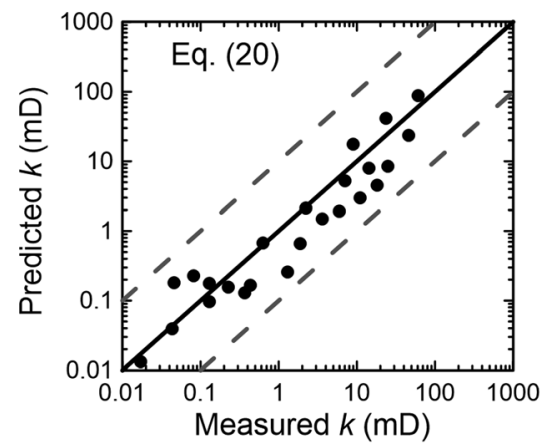

Figure 3. Comparison between measured permeability and model predictions. KC stands for the classical Kozeny-Carman model. The experimental data are from Zhang and Weller (2014). The coefficient of determination $R^{2}$ of $\log _{10}(k)$ is 0.895 in (a) and 0.933 in (b). The left panel corresponds to the prediction of the KC model, while the right panel corresponds to the prediction of the new model corresponding to equation (20).

As long as the information of media texture $\left(\phi, D_{f}\right.$, and $\left.S_{\mathrm{V}}\right)$ and bulk conduction $m$ is known, both permeability and surface conductivity can be predicted. Moreover, if the bulk and surface conductivities are measured in practice, the pore geometry information $D_{f}$ can be determined. As a side note, surface conductivity can be determined independently through induced polarization measurements (Niu et al., 2016), it is therefore possible to use combined conductivity and induced polarization measurements to determine permeability.

\section{Conclusions}

Archie's law characterizes the relationship between the bulk conductivity to porosity and is characterized by a power law exponent greater than 1. Similarly, surface conductivity can be related to the specific surface area through a power law relationship that we call surface or interfacial Archie's law, which is characterized by a power law exponent smaller than 1. An analytical relation exists between the power law exponents entering the bulk and surface Archie's laws and the fractal dimension $D_{f}$ of the pore network. Just as the coefficient $a$ in the classical Archie's law should be physically set to a constant, the coefficient $a_{\mathrm{S}}$ in surface Archie's law also tends to some fixed value, at least for the fractals and cores under study. The predictions of surface Archie's law agree well with the simulations of conduction in synthetic materials characterized by a fractal dimension and with laboratory experimental data. A new permeability model is proposed, extending the KC permeability model. This model is successfully tested against experimental data.

\section{Appendix A: Surface Archie's Law for Fractal Porous Media With Contiguous Grains}

In this appendix, we consider the conductivity of a porous material characterized by fractional dimension and with some grain contiguity. From Sections2.2, we know that both the surface area and conductivity of self-similar surfaces decrease at constant rate, and their relationship satisfies Archie's law. The decreasing factors of the surface area and conductivity are written as $q_{\mathrm{S}}$ and $q_{\mathrm{C}}$, respectively, when more grains are added to the pore space. As a fractal at stage $i$ is comprised of $N$ fractals at stage $i-1$ being scaled $p$ times smaller, its inner surface can be divided into two parts: one includes the surface of the $N$ fractals at stage $i-1$; another includes the surface of the grains added at stage 1 , but multiplied by an area factor $q_{S}^{i-1}$. Then, the specific surface area at stage $i$ is

$$
\left(S_{\mathrm{V}}\right)_{i}=\frac{N}{p^{D-1}}\left(S_{\mathrm{V}}\right)_{i-1}+\frac{1}{q_{\mathrm{S}}^{i-1}}\left(S_{V}\right)_{1}
$$

and the conductivity is

$$
\sigma_{i}=\sigma_{i-1} f(1, G)+\frac{1}{q_{\mathrm{C}}^{i-1}} \frac{\Sigma_{\mathrm{S}}}{\left(F_{\mathrm{S}}\right)_{1}}
$$




$$
\sigma_{i}=\left(\frac{\sigma_{\mathrm{w}}}{F_{i-1}}+\frac{1}{q_{\mathrm{C}}^{i-2}} \frac{p \Sigma_{\mathrm{S}}}{\left(F_{\mathrm{S}}\right)_{i-1}}\right) f(1, G)+\frac{1}{q_{\mathrm{C}}^{i-1}} \frac{\Sigma_{\mathrm{S}}}{\left(F_{\mathrm{S}}\right)_{1}}
$$

Following the same derivation as in section $2.3, \sigma_{i}$ and $\left(S_{\mathrm{V}}\right)_{i}$ satisfy a surface Archie's law.

\section{Acknowledgments}

$\mathrm{H}$. Wang warmly thanks for the fruitful discussions with Dr. Peng Shi. This work is supported by Open Fund of State Key Laboratory of Oil and Gas Reservoir Geology and Exploitation (Chengdu University of Technology) (PLC20190406). The data from literatures can be obtained in Waxman and Smits (1968), Vinegar and Waxman (1984) and Zhang and Weller (2014). The simulation data are provided in the supporting information and are available (Wang \& Hongtao, 2020 , simulation and core data on surface conductivity and permeability, figshare. Dataset. https://doi.org/ 10.6084/m9.figshare.11860224.v2). We thank the Editor, one anonymous referee, and Dr. Paul Glover for useful discussions.

\section{References}

Allouche, J.-P., \& Shallit, J. (2003). The Sierpiński carpet. In Automatic sequences: theory, applications, generalizations, (pp. 405-407) Cambridge, England: Cambridge University Press.

Anovitz, L. M., \& Cole, D. R. (2015). Characterization and analysis of porosity and pore structures. Reviews in Mineralogy and Geochemistry, 80(1), 61-164. https://doi.org/10.2138/rmg.2015.80.04

Archie, G. E. (1942). The electrical resistivity log as an aid in determining some reservoir characteristics. Transactions of AIME, 146(01), 54-62. https://doi.org/10.2118/942054-G

Bernabé, Y., \& Bruderer, C. (1998). Effect of the variance of pore size distribution on the transport properties of heterogeneous networks. Journal of Geophysical Research, 513-525. https://doi.org/10.1029/97JB02486

Bernabé, Y., \& Revil, A. (1995). Pore-scale heterogeneity, energy dissipation and the transport properties of rocks. Geophysical Research Letters, 22(12), 1529-1532. https://doi.org/10.1029/95GL01418

Bussian, A. E. (1983). Electrical conductance in a porous medium. Geophysics, 48, 1258-1268.

Cai, J., Wei, W., Hu, X., \& Wood, D. (2017). Electrical conductivity models in saturated porous media: A review. Earth-Science Reviews, 419-433. https://doi.org/10.1016/j.earscirev.2017.06.013

Glover, P. W. J. (2016). Archie's law-A reappraisal. Solid Earth, 7(4), 1157-1169. https://doi.org/10.5194/se-2016-47

Glover, P. W. J., Hole, M. J., \& Pous, J. (2000). A modified Archie's law for two conducting phases. Earth and Planetary Science Letters, 180(3-4), 369-383. https://doi.org/10.1016/S0012-821X(00)00168-0

Hunt, A. G. (2004). Continuum percolation theory and Archie's law. Geophysical Research Letters, 31, L19503. https://doi.org/10.1029/ 2004GL020817

Johnson, D. L., Koplik, J., \& Schwartz, L. M. (1986). New pore-size parameter characterizing transport in porous media. Physical Review Letters, 57(20), 2564-2567. https://doi.org/10.1103/PhysRevLett.57.2564

Katz, A. J., \& Thompson, A. H. (1985). Fractal sandstone pores: Implications for conductivity and pore formation. Physical Review Letters, 54(12), 1325-1328. https://doi.org/10.1103/PhysRevLett.54.1325

Mendelson, K. S., \& Cohen, M. H. (1982). The effect of grain anisotropy on the electrical properties of sedimentary rocks. Geophysics, 47(2), 257-263. https://doi.org/10.1190/1.1441332

Niu, Q., Revil, A., \& Saidian, M. (2016). Salinity dependence of the complex surface conductivity of the Portland sandstone. Geophysics, 81(2). https://doi.org/10.1190/geo2015-0426.1

Patnode, H. W., \& Wyllie, M. R. (1950). The presence of conductive solids in reservoir rocks as a factor in electric log interpretation. Journal of Petroleum Technology, 2(02), 47-52. https://doi.org/10.2118/950047-G

Peitgen, H. O., \& Saupe, D. (1988). The science of fractal images. Verlag: Springer.

Revil, A., \& Cathles, L. M. (1999). Permeability of shaly sands. Water Resources Research, 35(3), 651-662. https://doi.org/10.1029/ 98WR02700

Revil, A., Murugesu, M., Prasad, M., \& Le Breton, M. (2017). Alteration of volcanic rocks: A new non-intrusive indicator based on induced polarization measurements. Journal of Volcanology and Geothermal Research, 341, 351-362. https://doi.org/10.1016/j. jvolgeores.2017.06.016

Roy, S., \& Tarafdar, S. (1997). Archie's law from a fractal model for porous rocks. Physical Review B, 55(13), 8038-8041. https://doi.org/ 10.1103/PhysRevB.55.8038

Sen, P. N., Scala, C., \& Cohen, M. H. (1981). A self-similar model for sedimentary-rocks with application to the dielectric-constant of fused glass-beads. Geophysics, 46(5), 781-795. https://doi.org/10.1190/1.1441215

Shabro, V., Kelly, S., Torres-Verdín, C., Sepehrnoori, K., \& Revil, A. (2014). Pore-scale modeling of electrical resistivity and permeability in FIB-SEM images of organic mudrock. Geophysics, 79(5), D289-D299. https://doi.org/10.1190/GEO2014-0141.1

Thompson, A. H., Katz, A., \& Krohn, C. E. (1987). The microgeometry and transport properties of sedimentary rock. Advances in Physics, 36(5), 625-694. https://doi.org/10.1080/00018738700101062

Vinegar, H. J., \& Waxman, M. H. (1984). Induced polarization of shaly sands. Geophysics, 49(8), 1267-1287. https://doi.org/10.1190/ 1.1441755

Walsh, J. B., \& Brace, W. F. (1984). The effect of pressure on porosity and the transport properties of rock. Journal of Geophysical Research, 9425-9431. https://doi.org/10.1029/JB089iB11p09425

Wang, H., \& Liu, T. (2017). Derivation of Archie's law based on a fractal pore volume. Geophysical Journal International, 209(3), 1403-1407. https://doi.org/10.1093/gji/ggx095

Waxman, M. H., \& Smits, L. J. M. (1968). Electrical conductivities in oil-bearing shaly sands. Society of Petroleum Engineers Journal, 8(02), 107-122. https://doi.org/10.2118/1863-A

Wei, W., Cai, J., Hu, X., \& Han, Q. (2015). An electrical conductivity model for fractal porous media. Geophysical Research Letters, 42, 4833-4840. https://doi.org/10.1002/2015GL064460

Winsauer, W. O., Shearin, H. M. Jr., Masson, P. H., \& Williams, M. (1952). Resistivity of brine-saturated sands in relation to pore geometry. AAPG Bulletin, 36(2), 253-277. https://doi.org/10.1306/3D9343F4-16B1-11D7-8645000102C1865D

Wong, P.-Z., Koplik, J., \& Tomanic, J. (1984). Conductivity and permeability of rocks. Physical Review B, 30(11), 6606-6614. https://doi.org/ 10.1103/PhysRevB.30.6606

Yue, W. (2019). Pore-scale explanation of the Archie's cementation exponent: Microstructure, electrical anisotropy, and numerical experiments. Geophysical Research Letters, 46, 5799-5807. https://doi.org/10.1029/2019GL082585

Zhang, Z., \& Weller, A. (2014). Fractal dimension of pore-space geometry of an Eocene sandstone formation. Geophysics, 79(6). https://doi.org/10.1190/geo2014-0143.1 